\title{
Ultra-low price of generic agents in China may weaken patients' drug recognition and compliance
}

\author{
Saihua $\mathrm{Lu}^{1}$, Jian $\mathrm{Cai}^{2}$, Feng $\mathrm{Xu}^{1, *}$ \\ ${ }^{1}$ Department of Clinical Pharmacy, Fengxian Hospital, Southern Medical University, Shanghai, China; \\ ${ }^{2}$ Department of Hospital Office, Fengxian Mental Health Center, Shanghai, China.
}

\begin{abstract}
SUMMARY Drug quantity purchase in China has reshaped the pattern of drug market with lower price of generic drugs and relatively higher price of original brand-name drugs. However, most Chinese people do not trust the safety and efficacy of the generic drugs that sold at a very low price. Ultralow price of generic agents may weaken patients' drug recognition and compliance, and affect the implementation of drug quantity purchase policy.
\end{abstract}

Keywords Drug quantity purchase, drug price, brand recognition, medication compliance

Under the spotlight, China's drug price negotiation was finally settled at the end of $2019(1,2)$. It is encouraging to see the results of drug pricing negotiations help patients reduce the economic burden. Drug quantity purchase policy in China is executing, and is to expand from its major $4+7$ cities to all over the country. The intention of the quantity purchase policy is to improve the medication accessibility for ordinary peoples, meanwhile to lower national healthcare costs and to contain the too fast rising medical expense. The generic drug advocation now is in full swing in this country since China Food and Drug Administration (CFDA) published Opinions on Evaluation of Consistency of Quality and Efficacy of Generic Drugs in 2016. CFDA originally scheduled to complete quality and efficacy consistency evaluation of 289 generic drugs with original brand name drugs in late 2018, however, since the workload is large and cost is high, it is an impossible mission.

Up to now, a few generic agents have passed the consistency evaluation of the quality and efficacy with original branded drugs, and are qualified for quantity purchase in the whole country. The centralized drugs quantity purchase project has significantly reduced the prices of drugs, which reshaped the pattern of drug market with lower price of generic drugs and relatively higher price of original branded drugs. For example, the price of original brand-name glimepiride (Amaryl, Sanofi Aventis) is $64 \mathrm{CNY}$ for $2 \mathrm{mg} \times 15$ tablets in China market, while the price is as low as only 0.0198 CNY (about 0.00283 USD) for a piece of generic counterpart tablet.

In order to push the implementation of policy, the
National Healthcare Security Administration (NHSA) requires that prescription priority should be given to generic drugs in hospitals of all levels. Will ultra-low price of generic agents obtain satisfaction from all levels of people? Based on our pilot interview with outpatients, we believe the situation perhaps is not optimistic.

During the interview, most patients claim that they sincerely believe in "the higher the price, the better the quality of the merchandise", "you get what you pay for". Therefore they do not trust the safety and efficacy of generic drugs that are sold at a very low price. Many patients express that they are rich and affordable to everything, not to mention "lifesaving medicine". In their opinions, price differences are usually used as a proxy for differences in quality. Generic drugs with too low price seem to be impossible to be with good quality. "Can you image that a drug as cheap as cabbage could cure your disease?" Some patients made such comments. The deep-rooted reason is related to consumer psychology. A few Chinese people worship and have blind faith in things foreign $(3,4)$. Furthermore, the most leisured class prefer to use the high price original brand-name drug just out of show-off psychology (5). Facing disease treatment and medicine choice, even low-income Chinese parents would choose original branded drugs for their sick baby and child in spite of emptying their purse (6). In fact, a strong brand loyalty has been built among ordinary Chinese people. It is difficulty to change the current situation.

There is still a long way to go to persuade patients to use extremely cheap generic drugs. In the generic 
drug advocate in China, we suggest that NHSA should guarantee appropriate profit for those generics instead of just blindly lowering the price of drugs. It is a truth universally acknowledged that appropriate profit is better than small profit and/or no profit to guarantee the quality of drugs. As the Chinese saying goes, "Cheap goods are not good, while good goods are not cheap". Generic drug with appropriate price might give patients a little more confidence, enhance patients' drug recognition and compliance than those dirt cheap counterparts.

\section{Acknowledgements}

This work was supported by the Medicine Guide Program of Shanghai Municipal Science and Technology Commission (grant no. 19411971700), Shanghai Municipal Clinical Pharmacy Key Discipline Reconstruction Program (grant no. 2018-2020). The authors declare that they have no conflicts of interest to this work.

\section{References}

1. Tang M, He JJ, Chen MX, Cong LX, Xu Y, Yang Y, Hou ZY, Song PP, Jin CL. "4+7" city drug volume-based purchasing and using pilot program in China and its impact. Drug Discov Ther. 2019; 13:365-369.

2. Tang M, Song PP, He JJ. Progress on drug pricing negotiations in China. Biosci Trends. 2019; 13:464-468.

3. Cai J, Ye M, Fei C, Xu F. Impact of brand-name drug worship and expectation psychology on antidepressant efficacy. Int J Clin Exp Med. 2013; 6:724-726.

4. Li M, Cai J, Zhang P, Fei C, Xu F. Drug brand response and its impact on compliance and efficacy in depression patients. Front Pharmacol. 2017; 7:540.

5. Hodgson GM. Darwin, Veblen and the problem of causality in economics. Hist Philos Life Sci. 2001; 23:385-423.

6. Xiao ZJ, Xu F. Health literacy and medication preference: Why do most patients choose imported brand-name drugs? Patient Educ Couns. 2014; 95:155156.

Received January 8, 2020; Revised March 25, 2020; Accepted April 14, 2020.

*Address correspondence to:

Dr. Feng Xu, Clinical Pharmacy, Fengxian Hospital, Southern Medical University, 6600 Nanfeng Hwy, Fengxian District, Shanghai 201400, China.

E-mail: xuf@smu.edu.cn

Released online in J-STAGE as advance publication April 15, 2020. 